\title{
INNOVATIVE DESIGN WITHIN TRADITION - INJECTING TOPOS STRUCTURES IN C-K THEORY TO MODEL CULINARY CREATION HERITAGE
}

\author{
Hatchuel, Armand; Le Masson, Pascal; Weil, Benoit; Carvajal-Perez, Daniel \\ MINES ParisTech-PSL
}

\begin{abstract}
In "Grande cuisine" creation and tradition co-evolve in a rich number of ways. Great chefs still use recipes from the 19th century and may also reinvent gastronomy itself. The creation heritage of culinary Art is the paradoxical capacity to both "respect" tradition and "break" its rules. Building on C-K theory, we show that such creative heritage needs multiple and independent layers of knowledge that "speak" of basic fixed objects. These properties correspond to general mathematical structures that we find in Topos theory. Thus, $\mathrm{C}-\mathrm{K} /$ Topos predicts creative design strategies that can respect tradition in different ways. It also proves a form of "innovation within tradition" - "sheafification" in Topos words- that is not a compromise and builds on tradition itself. These findings fit with the lessons of great books of gastronomy. C-K/Topos has a wide scope of validity: it applies to any innovative design that needs preserving systemic structures, like engineering systems or social and environmental systems. C$\mathrm{K} /$ Topos models with a high generality how local and radical innovation can warrant systems incremental change. $\mathrm{C}-\mathrm{K} /$ Topos will have implications for teaching and research.
\end{abstract}

Keywords: Creation heritage, Tradition, Innovation, Design theory, Knowledge management

Contact:

Le Masson, Pascal

MINES ParisTech-PSL

Management Science

France

pascal.le_masson@mines-paristech.fr

Cite this article: Hatchuel, A., Le Masson, P., Weil, B., Carvajal-Perez, D. (2019) 'Innovative Design Within Tradition Injecting Topos Structures in C-K Theory to Model Culinary Creation Heritage', in Proceedings of the 22nd International Conference on Engineering Design (ICED19), Delft, The Netherlands, 5-8 August 2019. DOI:10.1017/dsi.2019.160 


\section{INTUITIVE MOTIVATION: THE CREATION HERITAGE OF CULINARY ART?}

What is the heritage of a creative craft? "Grande cuisine" is one of the most iconic crafts where creation and tradition co-evolve in a rich number of ways. Great chefs still use common food ingredients and classical recipes already described in Cuisine books of the 19th century. Other chefs have reinvented meals, culinary techniques and redefined gastronomy (Agogué and Hatchuel 2016) or use design methodologies to foster creativity (Capdevila et al., 2015) while . Actually, culinary Art have long been the subject of important transmission efforts. They gave rise to famous "grande cuisine" treatises that had a major impact on the creative development of the craft. Intuitively, we are facing a paradoxical notion: the heritage of culinary Art appears as a "creation heritage" which is a capacity to both: i) "respect" a craft tradition; and ii) "break" traditional rules and regenerate the tradition! Most natural view is rather that tradition and innovation are contradictory or that a creation heritage only teaches designs compromises between tradition and innovation. It is more difficult to think that tradition could guide and foster innovation. Therefore, Culinary art challenges our views about tradition and innovation. It also offers a good empirical reference for studying the enigma of a "creation heritage": what type of knowledge can contain both fixed rules to be respected and a creative potential? The purpose of this research is to establish a formal description of the structure, logic and conditions of a creation heritage. In this paper we explore, with the perspective of $\mathrm{C}-\mathrm{K}$ theory, how such creative heritage can correspond to special mathematical structures that help modelling the "respect" of tradition in a richer way; and reveal forms of innovation that have their source in tradition itself. Our research assumes that the culinary creation heritage has been encapsulated in influential books that have been landmarks in the history of gastronomy. However, beyond culinary art, the model developed in this paper has a much larger scope of validity that we highlight in our conclusion.

Research methodology: modelling a creation heritage with $\mathbf{C}-\mathrm{K}$ theory. To elaborate a general model that captures a creation heritage and its forms of creative design, we have followed different steps that are developed in the sections of this paper.

a) We build on $\mathrm{C}-\mathrm{K}$ theory as a universal model for creative design (Hatchuel and Weil 2003; Hatchuel and Weil 2009). C-K theory defines a family of models $\mathbf{C}-\mathbf{K} / \mathbf{X}$ where $\mathbf{X}$ is a specific knowledge space. The structure of $\mathrm{X}$, determines a corresponding creative capacity or design regime (Le Masson et al., 2017). Hence, our research question becomes: what are the structures of $X$ such that $C-K / X$ describes a creation heritage that also respects tradition?

b) From analysis and the study of culinary books, we first establish that a creative heritage including tradition follows a canonical model of the form $\mathbf{C}-\mathbf{K} / \mathbf{K n h}(\mathbf{K n}-\mathbf{1 h}(\ldots(\mathbf{K 1 h}))))$ where Kih are layers of knowledge such that : i) no layer Kih determines another one; and ii) Traditional objects common to all Kih remain unchanged by design. Thus, our new question: is there a good mathematical interpretation of such canonical model and what can it tell us about $\mathrm{CH}$ ?

c) We found that the mathematical structures of a Topos mirror the canonical model of CH. Topos theory studies with a high generality, universes of objects and relations that require several levels of description: for instance : i) different logics built on the same geometric space ; or ii) global and local properties of a universe. In this section, we give an elementary introduction to Topos notions and we show that C-K/Topos captures all paradoxical aspects of a creation heritage and reveals how tradition can be respected and still guide creative design strategies. Topos allows modelling "innovation within tradition" in a rigorous and general way.

\section{PART 1: A CREATION HERITAGE: LAYERS OF KNOWLEDGE, GENERATIVITY AND TRADITION}

\subsection{Beyond know-how: A creation heritage as a generative heritage}

The first component of a creation heritage $\mathrm{CH}$ is a list of know-how propositions Klh easily taught to a person. In Gastronomy, it is usually a description of cooking recipes. However, we know from C-K theory (Hatchuel et al., 2018; Hatchuel et al., 2013b; Hatchuel et al., 2011a) that new designs are 
possible only if $\mathrm{K} 1 \mathrm{~h}$ verifies universal conditions for generativity (UG conditions) ${ }^{1}$. Obviously, a pure list of cooking recipes or a "lego type" recipes - common to cooking books - do not fulfil UG conditions and $\mathbf{C}-\mathbf{K} / \mathbf{K} \mathbf{1 h}$ is not a creation heritage.

Consequently, a creation heritage needs another layer of knowledge K2h that fulfils UG conditions. Moreover, K2h cannot be deduced from K1h otherwise K2h would be part of K1h. Conversely, K1h cannot be deduced from $\mathrm{K} 2 \mathrm{~h}$ : if this was true, $\mathrm{K} 2 \mathrm{~h}$ would be itself a know-how that does not fulfil UG conditions. Thus the creation heritage is at least of the form $\mathbf{C}-\mathbf{K} / \mathbf{K} 2 \mathbf{h}(\mathbf{K} 1 \mathbf{h})$. the symbol $\mathrm{K} 2 \mathrm{~h}(\mathrm{~K} 1 \mathrm{~h})$ means a structure of knowledge where the layer K2h "speaks about" objects of K1h without constituting a proposition of K1h.

Example: culinary books foster generativity, for instance, by associating new qualities to a meal without giving the new recipe. In El Bullis's manifesto "Synthesis of elBulli cuisine" (see appendix), the great chef Ferran Adria states that "Cooking is a language through which all the following properties may be expressed: harmony, creativity, happiness, beauty, poetry, complexity, magic, humour, provocation and culture". Thus any classic recipes, menus, dressings, aesthetics of the tradition can be revisited and new concepts generated like "a provocative tortilla". But $\mathrm{CH}$ must also include the respect of tradition.

\subsection{Tradition heritage as a regulatory knowledge}

A creation heritage needs also to define the content of tradition ${ }^{2}$. One role of tradition is to limit the capacity to change existing know-how. Hence, the creation heritage includes a third level of knowledge K3h that controls if some creative design respects the craft tradition. $\mathbf{K 3 h}$ is a regulatory knowledge that "tunes" the generative power of $\mathrm{C}-\mathrm{K} / \mathrm{K} 2 \mathrm{~h}(\mathrm{~K} 1 \mathrm{~h})$. Hence the existence of a craft tradition implies that a creation heritage is of the form $\mathbf{C}-\mathbf{K} / \mathbf{K} 3 \mathbf{h}(\mathbf{K} 2 \mathbf{h}(\mathbf{K} 1 \mathbf{h})))$. K3h can be also a generator of design strategies that helps balancing between tradition and innovation in different ways. In the domain of Culinary art, we can observe design strategies where tradition is maintained partially. For example, redesigning a classic French recipe (tradition) with the purpose of adding freshness \{Hatchuel, $2009 \# 1549\}$ or fatless diet.

\subsection{Canonical model of a creation heritage: Generativity, independences and respect of tradition}

The series $\mathrm{K} 1 \mathrm{~h}, \mathrm{~K} 2 \mathrm{~h}, \mathrm{~K} 3 \mathrm{~h}$ is a special case of a more general logic. The balance between tradition and innovation can be itself regulated by another level of knowledge K4h that changes that balance by associating new references (Art, fashion..) or new cooking cultures (European, asian, African..). For the sake of generality, we need assuming a variable number if of knowledge layers: $\mathbf{K} \mathbf{1 h}, \mathbf{K} \mathbf{2} \mathbf{h}, .$. , Knh... Then the canonical form of a creation heritage can be finally defined as: $\mathbf{C H}=\mathbf{C}-\mathbf{K} / \mathbf{( K n h}(\mathbf{K n}-$ $\mathbf{1 h}(\mathbf{K n}-\mathbf{2 h}) \ldots(\mathbf{K} \mathbf{h})))))$ if the following general properties of the Kihs are assumed:

- Generativity: At least one layer Kih fulfils the UG conditions

- Independences: no layer can be deduced from any other: this means that each layer brings independent information.

- respect of tradition: All layers of knowledge can evolve, yet there exists a basic structure of objects and rules that is common to all layers and will serve to define what means "respecting" tradition.

Examples. Great culinary books offer numerous illustrations of such canonical model. As predicted, they all are organized in independent different layers of knowledge. For example, in famous books (Dubois and Bernard 1856; Escoffier 1902), funds and sauces are both considered as the core of the art

\footnotetext{
${ }^{1}$ Generativity conditions are: expandability, existence of concepts ("holes of undecidability"), splitting condition (Le Masson et al. 2016) allowing new designs from concepts (Hatchuel et al. 2011a; Hatchuel et al. 2013a)

${ }^{2}$ If complete freedom of design exists there is no tradition to be respected. This situation corresponds to highly innovative domains i.e. when creative design can lead to complete changes in the identity of the techniques and objects of the domain (Le Masson et al. 2016). Examples exist in engineering (mechanical engines can be replaced by electric ones; analogic music can be replaced by digital sounds, robots can replace drivers...). Contemporary forms of Art are also good examples of free creation heritage.
} 
and also a space for new designs. Funds and sauces are not complete meals recipes. They form a special layer of knowledge and the use of sauce in recipes is both traditional for some meals but a same sauce can be associated to a large variety of recipes and meals. The same logic appears with « croquettes » which can «have infinitely different forms and compositions» (Dubois and Bernard 1856). Above recipes, funds and sauces, another layer of knowledge informs about «well composed menus » (series of different meals) which should be adapted to circumstances and clients. They have to express «a sure design, a high talent, and well balanced spirit that masters all the resources of culinary art » (Dubois and Bernard 1856). Ornaments introduce another layer of knowledge. For a good ornament Escoffier indicates that: "to reach this result, the inventive worker has numerous available means: using only edible elements like truffles, mushrooms, egg whites, vegetables, tongue, etc. , she can combine an infinite variety of admirable ornaments". In this case, Escoffier fixes the tradition "ornaments should be edible" and opens for generative design.

As indicated above, all these levels of knowledge clearly do not determine each other and do not lead to a unique set of cooking recipes. Menu are composed of meals, but the composition of meals is not fully determined by the menu. Ornaments depend of the meals but also of the context of the menu. Thus the canonical model of a creation heritage is clearly not a fixed set of fabrication and assembly rules. Each layer of knowledge introduces new objects and new relations. Each one organizes a specific perspective about the universe of recipes, with its own share of tradition and space for free creation. All these levels of knowledge are also interrelated; and they all, in their own way, «speak» of the same basic objects. They form a creation heritage and confirm the canonical model introduced before.

However, this canonical model raises several technical questions. What allows tradition and creative design to co-evolve without creating contradictions or destroying the craft's unity? In this process, is innovation just "constrained" or is there a new form of innovation that is related to a creation heritage? To explore and answer rigorously these questions, we need a formalized representation. Thus, our research question can be now expressed more technically: can we find a mathematical model that mirrors such multilayer structure and its three specific properties (generativity, independence, and tradition)? If such model exists, what does it tell us about innovative design within such structures?

\section{PART 2. C-K THEORY AND TOPOS THEORY: MODELLING INNOVATION WITHIN TRADITION}

There is a long history of knowledge representation. The first tradition developed after Herbert Simon described knowledge as sets of rules. Later, knowledge was described with ontologies or Type models. In mathematics, the last decades assessed the generality, richness and power of Topos theory which introduces very flexible representations of knowledge that can cope with global and local structures of knowledge. Topos also can combine in a unique and general way both space properties and logics. Topos appeared as the best candidate to formalize the canonical model of $\mathrm{CH}$ and its properties for the following reasons:

1. Topos can be built on one fixed category

2. Topos capture universes that present layers of information and are too complex to be described by Sets and standard logic (Prouté 2007))

3. Topos have a generative power. It was shown that the technique of 'forcing' (Cohen 1963) ie. a design of new models, can be generalized in Topos theory (Tierney 1972). Hence C-K/Topos fulfills the UG conditions.

Now we establish the close correspondence between C-K/ Topos and a creation heritage. In this section we give elementary notions about Topos theory focusing on those which are relevant to the formalization of a creation heritage. In the following, we describe the interpretations that we associate to the building category $\mathbf{C}$, presheafs $\mathbf{P S h}$, subobject classifiers $\mathbf{\Omega}$, Sites $\mathbf{S}$, and Sheafs $\mathbf{S h}$.

\subsection{The building category $\mathrm{C}$ and its presheafs}

The building Category $\mathrm{C}$ represents a first layer of knowledge about objects and relations of the Topos. It corresponds to the basic knowledge that defines the tradition of the craft. For Culinary art, $\mathrm{C}$ describes the main definitions of ingredients, meals, menus, recipes, techniques and habits that compose the common knowledge of the Craft. For modern cuisine the famous Escoffier Book is a good inventory of that knowledge. It can be seen as the Know-how Klh that any chef should know, even the most innovative ones like Ferran Adria (El bulli's manifesto). 
A Topos is not limited to $\mathrm{C}$ but contains all applications $\mathrm{F}(\mathrm{C})$, called presheaves on $C$ (Psh (C)) from $\mathrm{C}$ to any set of values. For instance, the Topos contains presheaves describing the "nutritional impact" of objects in C. or their "gastronomic values". Metaphorically, the category C plays the role of a basic "geometry" or "space" in which one 'describes' some new aspects than C itself. To one unique category $\mathrm{C}$ will correspond many presheaves, i.e. many layers of knowledge which share a reference to C: they "speak" about C. The structure of a Topos can be represented by the figure below (Kostecki 2011)).

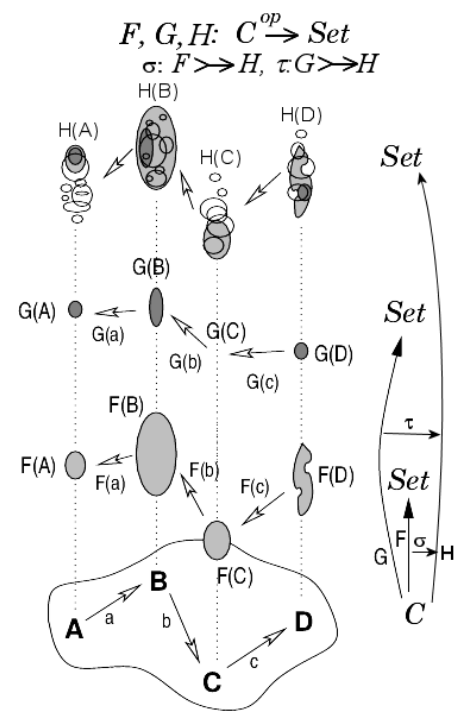

Figure 1. (taken from (Kostecki 2011)): A category of pressheaves. The category $C$ has the objects $A, B, C$ and $D$ and the arrows a, b, $c$ (and the compositions); $F, G$ and $H$ are presheaves of sets on $C$.

Proposition. If presheafs are independent, a Topos has naturally the multilayer structure of the canonical model of $\mathrm{CH}$.

\subsection{Subobject classifier of a Topos: Modelling the laws of tradition}

Moreover, in Topos structures, the consistency of tradition is warranted by the stability of $\mathrm{C}$ and by its internal logic. This logic is precisely described by a central notion of any Topos: the subobject classifier that we introduce by an example.

Let us assume that a chef has to select the dishes that are adapted to "a luxury dinner", he will build a presheaf on C ( an application of C on some Kih) - that we call "Lux" - which is also a subobject of the Culinary Topos. To be consistent with $\mathrm{C}$, the entities of Lux have to be "dishes": i.e. they must respect the structure of dishes in $\mathrm{C}$. Assume that in $\mathrm{C}$, all dishes are composed of a meat and sauce and a meat never goes without sauce. Hence, Lux is necessarily composed of only three classes of objects: Dishes that are in Lux with meat and sauce, Dishes that are not in Lux, Dishes that are in Lux but only with sauce (a sauce that makes lux in itself). Note that the classic set theory answer would be "dishes that are, or are not in Lux". The structure of C, the cooking tradition, appears in the existence of the third possibility: dishes that are in Lux but only with sauce. Let's underline that this richer logic of the topos comes from the interdependence between objects fixed in C. Moreover, it is a theorem of Topos theory, that :

- the composition \{sauce $\rightarrow$ meat $\rightarrow$ dish\} will impact all presheafs in the Topos i.e. all subobjects of the Topos and

- this impact is modelled by a unique subjobject classifier which only contains the three different classes that we have mentioned as they serve to build a subobject of a Topos.

Thus a Topos describes correctly a universe of objects that are built on the same stable tradition C.

Proposition: a Topos is a good model of a creation heritage as all presheafs of the Topos respect the logic of the building category i.e. the traditional know-how of the craft. 


\subsection{The generative capacity of C-K/Topos: Concepts as "presheafs that are not sheaves"; Sites as ways to respect tradition}

We have now to show how some creative design is possible in a Topos based design theory i.e. in C$\mathrm{K} / \mathrm{Topos}$. In the previous example, what happens if we attempt to design "vegetarian dishes that are in Lux"? This formulation builds a presheaf on $\mathrm{C}$ but we cannot specify the value of this presheaf for all objects of $\mathrm{C}$ (since there is no specification for the meat!) i.e. there is no class in the subject classifier that contains such objects. We need to introduce the notion of sheaf that can be defined as a "well formed" presheaf. In Topos theory, a presheaf is "well-formed" (and is then called a sheaf) if the presheaf application preserves specific structures $\mathrm{J}$ of objects of $\mathrm{C}$ (note that $\mathrm{J}$ is itself in $\mathrm{C}$ and is, more precisely a specific subobject of $\Omega$ ). These specific types of subobjects $\mathbf{J}$ are called (Grothendieck) "topologies" and the pair $(\mathrm{C}, \mathrm{J})$ is called a site.

Topos theory proves that in a given category $\mathrm{C}$, with its given sub-object classifier $\Omega$, there are many such (Grothendieck) "topologies" J, i.e. many sites (C, J). As described below, J defines the "preservation" level for a given tradition C - hence it means that there are different "levels of requirement" in the preservation of tradition. Thus transforming a presheaf (eg "vegetarian lux") into a sheaf on $(\mathrm{C}, \mathrm{J})$, means obtaining a complete design that respects $\mathrm{C}$ "in the $\mathrm{J}$ way". It corresponds to the transformation of an unknown concept into a known object in $\mathrm{C}-\mathrm{K}$ theory, with the specific situation that one wants to get a final object that respects $\mathrm{C}$ in the $\mathrm{J}$-sense. Thus Topos theory provides us with a clear description of how "respect of tradition" can be built in different ways that all belong to tradition.

Finally, Topos theory predicts that the design of "a vegetarian dish that is in Lux" can be done in two different ways: by classic design strategies (adding knowledge and adapting the category ) or by "sheafification" i.e.by innovation within tradition. Let's illustrate these two strategies in the case of the "vegetarian dish".

\subsection{Classic design strategies: A "compromise" between tradition and innovation}

Consider the "vegetarian dish that belongs to Lux". Three options are possible which correspond to usual "tuning" strategies between tradition and creation":

- Orthodoxy: the site $\mathrm{J}$ is "demanding" (this is the so-called "coarse" topology), which means that the presheaf application is only applied to "completely defined" subobjects - hence it is impossible to speak of a vegetarian dish without fully describing the sauce and the meat. Since there is no meat in a vegetarian dish, The coarse topology corresponds to a strategy of tradition preservation that will explain that a "vegetarian dish" is false, i.e. can't exist in this particular $(\mathrm{C}, \mathrm{J})$ : in this particular tradition and with this preservation strategy. With this $(\mathrm{C}, \mathrm{J}$ ) (where $\mathrm{J}$ is coarse) the tradition preservation strategy impedes innovation.

- Local convention - nominalism: the site $J$ is now "less demanding" (it is for instance a 'dense' topology) - J allows to describe a dish "incompletely". It can be said that J speaks of a dish "from the point of view" of its sauce, without necessarily describing its meat. Still to be well-formed, a pressheaf should enable to clearly identify all the subojects, and in particular the meat, even when the meat was undefined initially. Hence the pressheaf should come with an additional rule that makes that it is possible to determine the "meat" of a vegetarian dish from the chosen sauce. Then the only possibility is to add a rule that says that the sauce of the vegetarian dish is a "type of meat". This is a 'local convention' in the sense that it locally extends the "meat" object by adding one (relatively) strange attribute: "vegetarian sauce can be called meat". Tradition is preserved, innovation is confined to a local extension.

- Innovation: Finally, we can change the category itself. We consider for instance a category where we say that a dish is characterized by the fact that there is a sauce. We forget about the meat. The scope of creation is now completely open and tradition is broken.

These three cases rejoin classical design strategies that illustrates the "compromise" between tradition and innovation. An important finding is that they can be systematically deduced from the structure of the Topos,

\footnotetext{
${ }^{3}$ Mathematically, these strategies correspond to the choice of Topology on $\mathrm{C}$ that is called a site. The
} sheafification depends of the site selected. 
Proposition: $C$-K/Topos models a creation heritage and also predicts classic design strategies that make a compromise between innovation and tradition.

\subsection{Innovation within tradition: How sheafification in a topos revises the definition of objects inside tradition}

Now, Topos theory explains and warrants a more interesting process that actually goes beyond the compromise between tradition and innovation. With this process it is actually possible to revise the definitions of objects inside a tradition and, even more, thanks to the tradition. This is the sheafification technique which at the core of Topos theory. It is not possible in this paper to present all mathematical aspects of sheafification. But there are important theorems that confirm that this operation corresponds to Cohen's Forcing within a Topos \{Lawvere, 1964 \#3730; Tierney, 1972 $\# 3376$ \}. And we already know that Forcing is equivalent to creative design in C-K theory \{Hatchuel, 2013 \#2620\}. Thus we can establish that in C-K/Topos, sheafification is well defined and describes a rigorous design strategy.

Mathematically, sheafification is a curious process that can be described as such: when a presheaf is not a sheaf in a given Site, it is possible to design a new 'associated' shief in the same Site. This new sheaf 'extends' the presheaf, which means that it 'contains' the presheaf but it is still a sheaf. Keeping the same site means that:

- i) it keeps the category, hence the tradition;

- ii) it keeps the topology, i.e. the preservation logic of tradition,

- iii) it controls the "expansion" logic, i.e. it creates a new definition of the objects of the tradition, and extends it while preserving it.

Example : in the case of the "vegetarian dish", the sheafification process will actually lead to define "a meat without meat" (and this goes beyond the "nominalist' strategy that just named 'meat' a certain sauce). This definition of "a meat without meat" will be made by redefining the notion of "meat" inside the category $\mathrm{C}$ while keeping its relations with all the other objects in the category (relation between meat and sauce and between meat and dish) - the process of redefinition of 'meat' requires to study the whole range of possible relationships with each subobject of the system (what does the sauce tells about possible 'meats' -accordance and complementarities in texture, in tastes, in temperatures,...-, what does the 'dish' says about possible 'meats' -general balance, colors, savours, nutriments, etc.-). In the end we can, for instance, redesign the 'meat' as the nutriments and proteins of the dish (in a dietetic tradition!). Note that one can prove that, to generate a new subobject (compatible with the category), the sheafification requires a site that follows the splitting condition (Le Masson et al., 2016).

Actually, one can hardly underestimate the paradoxical generative power of sheafification:

- it changes the definition of (sub)-objects of the tradition but keeps the tradition! Hence it is radical innovation still in tradition

- the design of the new identity is actually fully based on the tradition. It is a "rule-breaking" innovation that is based on the rules of the tradition.

- it is a "defixation" (in the sense that it doesn't follow the definition of the objects) that is based on the fixation rules.

Finally, with the sheafification process, C-K/ Topos combines design and tradition in a way that is not a compromise. It allows a new form of innovative design (based on a strong renewal of the definition of objects) that keeps the tradition and is enabled by the tradition.

\section{DISCUSSION AND CONCLUSION}

\subsection{The impact of knowledge structures: Building and teaching a "creation heritage" for designers}

The scientific aim of Design theory is to offer a language and models that improve our understanding and provide new methods for teaching or for research. Intuition and common sense tend to oppose tradition and innovation. Our first finding is that the relation between tradition and innovation is much richer and is dependent of two parameters that have been revealed by this research: 
i) The structure of knowledge that is associated to tradition: we have seen that tradition contains a fixed set of objects and rules but that the heritage of a craft is not reducible to this unique layer of knowledge. It contains also several layers of knowledge that organize independence and prepare a generative capacity: tradition can contain knowledge about how tradition can change and so on...

ii) a Topos model of knowledge warrants that such generative capacity "respects" a fixed set of objects. It also offers different definitions of "respect" (sites), and some of these definitions allow the existence of an unexpected form of "innovation within tradition", technically modelled by the "sheafification" process.

Consequently, we can rigorously define now with great generality what is a "creation heritage" for designers. Clearly, it is more than a set of past designs, or a fixed set of objects and rules. It has to add to those memories, several other layers of knowledge that prepare generativity and it has to train students to both: i) classic design strategies and ii) to innovation within tradition i.e. reinventing the identity of objects within tradition.

This finding confirms that learning design by projects needs a thorough methodological control (Hatchuel et al., 2011b): we have at least to check which design strategies are learned in projects and if necessary, create additional training to teach missing design strategies.

\subsection{Beyond Culinary Art: Innovation within tradition as a universal design strategy in systems}

The introduction of sophisticated mathematical instruments, like Topos theory requires obust justification. First: we have argued that tradition, heritage, creative design are complex realities in culinary art. Second: such complexity is confirmed by the text books of grand chefs. Third: to overcome complexity we need understandable models which prove our findings, are sufficiently general, and have clear assumptions. This is the traditional role of mathematical models in any science. A specific difficulty in Design science is that the mathematical models which are relevant are not classic ones and do not belong to usual maths for engineers. Hopefully, the findings obtained are worth the effort and we conclude by a brief summary of the important consequences of our findings which also indicate directions for further research.

a) (Hatchuel et al., 2013b) already introduced the idea that C-K theory could be seen as a generalization of Forcing to real objects. Actually, the sheafification process generalizes Forcing to universes that are no more Sets. Thus, C-K/Topos appears as a powerful advance in Design theory which is extended to the wide class of objects that are defined by a category $\mathrm{C}$ and by all applications from this category to Sets.

b) The whole word of engineered or technical objects can be described by a Topos and all our findings about Culinary Art can be generalized to the world of technical objects. So, what is sheafification in engineering? Technically, sheafification creates a new object that is top-down and bottom up-compatible. This means that it fits with those objects that are dependent of its existence and with those objects that conditions its existence. In engineering terms, this means that interchangeability is warranted towards upper and lower components.

c) Such interchangeability is strategic and relevant for:

- - Engineering systems where innovation on one part of the system should not destabilize the system itself.

- - solving the classic opposition between incremental and radical innovation. Sheafification mixes the two notions: the new design is radically different from the old one (creating a vegetarian meat that is now a perfect meat in itself ) but the change appears incremental from the point of view of the design heritage (or the system).

d) Finally, studying how innovation can preserve tradition with the highest generality, we have built a model that can be applied to any preservation strategy. The social and environmental implications of such finding are of great importance. We have proved that sheafification techniques are necessary to any design strategies that aim to preserve social and environmental systems. The good news is also that radical innovation is possible even if society and nature have to be preserved! Yet, this achievement depends on our capacity to build knowledge structures about these systems that fulfil 
all the properties of a creation heritage. Fortunately, thanks to topos theory, these conditions are now rigorously understood and established.

\section{REFERENCES}

Agogué, M. and Hatchuel, A. (2016), "Reinventing classics: the hidden design strategies of renowned chefs", Research in Engineering Design, pp. 1-13.

Capdevila, I., Cohendet, P. and Simon, L. (2015), "Establishing New Codes for Creativity through Haute Cuisine". The Case of Ferran Adrià and elBulli. Technology Innovation Management Review Vol. 5 No. 7 , pp. 25-33.

Cohen, P. (1963), "The independence of the Continuum Hypothesis", Proceedings of the National Academy of Science Vol. 50, pp. 1143-1148.

Dubois, U. and Bernard, E. (1856), La Cuisine Classique. chez les auteurs, Paris

Escoffier, A. (1902), Le Guide Culinaire. 1902, 1907, 1912, 1921 edn. Flammarion, Paris

Hatchuel, A., Le Masson, P., Reich, Y. and Subrahmanian, E. (2018), "Design theory: a foundation of a new paradigm for design science and engineering", Research in Engineering Design Vol. 29, pp. 5-21.

Hatchuel, A., Le Masson, P., Reich, Y. and Weil, B. (2011a), "A systematic approach of design theories using generativeness and robustness", In: International Conference on Engineering Design, ICED'11, Copenhagen, Technical University of Denmark, 2011a. p 12

Hatchuel, A., Le Masson, P. and Weil, B. (2011b), "Teaching Innovative Design Reasoning: How C-K Theory Can Help to Overcome Fixation Effect", Artificial Intelligence for Engineering Design, Analysis and Manufacturing, Vol. 25 No. 1, pp. 77-92.

Hatchuel, A., Reich, Y., Le Masson, P., Weil, B. and Kazakçi, A.O. (2013a), "Beyond Models and Decisions: Situating Design through generative functions", Paper presented at the International Conference on Engineering Design, ICED'13, Séoul, Korea,

Hatchuel, A. and Weil, B. (2003), "A new approach to innovative design: an introduction to C-K theory", In: ICED’03, August 2003, Stockholm, Sweden, 2003. p 14

Hatchuel, A. and Weil, B. (2009), "C-K design theory: an advanced formulation". Research in Engineering Design Vol. 19 No. 4, pp. 181-192.

Hatchuel, A., Weil, B. and Le Masson, P. (2013b) "Towards an ontology of design: lessons from C-K Design theory and Forcing", Research in Engineering Design, Vol. 24 No. 2, pp. 147-163.

Kostecki, R.P. (2011), An Introduction to Topos Theory, Institute of Theoretical Physics, Warsaw

Lawvere, F.W. (1964), "An elementary Theory of the Category of sets", Proceedings of the National Academy of Sciences Vol. 52 No. 6, pp. 1506-1511.

Le Masson, P., Hatchuel, A. and Weil, B. (2016), "Design theory at Bauhaus: teaching "splitting" knowledge". Research in Engineering Design, Vol. 27 No. April 2016, pp. 91-115.

Le Masson, P., Weil, B. and Hatchuel, A. (2017), Design Theory - Methods and Organization for Innovation. Springer Nature. https://doi.org/10.1007/978-3-319-50277-9

Prouté, A. (2007), "Sur quelques liens entre théorie des topos et théorie de la démonstration", Paper presented at the Conférence faite à l'Université de la Méditerrannée le 29 mai 2007.

Tierney, M. (1972), "Sheaf theory and the continuum hypothesis", In: Lawvere F.W. (ed) Toposes, algebraic geometry and logic. Springer Verlag, Heidelberg, pp 13-42

\section{APPENDIX: EL BULLI MANIFESTO}

In the mid-1990s a new style of cuisine began to be forged. Today, this style has been wholly consolidated and may be defined in the following terms:

1. Cooking is a language through which all the following properties may be expressed: harmony, creativity, happiness, beauty, poetry, complexity, magic, humour, provocation and culture.

2. The use of top quality products and technical knowledge to prepare them properly are taken for granted.

3. All products have the same gastronomic value, regardless of their price.

4. Preference is given to vegetables and seafood, with a key role also being played by dairy products, nuts and other products that make up a light form of cooking. In recent years red meat and large cuts of poultry have been very sparingly used.

5. Although the characteristics of the products may be modified (temperature, texture, shape, etc.), the aim is always to preserve the purity of their original flavour, except for processes that call for long cooking or seek the nuances of particular reactions such as the Maillard reaction.

6. Cooking techniques, both classic and modern, are a heritage that the cook has to know how to exploit to the maximum.

7. As has occurred in most fields of human evolution down the ages, new technologies are a resource for the progress of cooking. 
8. The family of stocks is being extended. Together with the classic ones, lighter stocks performing an identical function are now being used (waters, broths, consommés, clarified vegetable juices, nut milk, etc.).

9. The information given off by a dish is enjoyed through the senses; it is also enjoyed and interpreted by reflection.

10. Taste is not the only sense that can be stimulated: touch can also be played with (contrasts of temperatures and textures), as well as smell, sight (colours, shapes, trompe d'oeil, etc.), whereby the five senses become one of the main points of reference in the creative cooking process.

11. The technique-concept search is the apex of the creative pyramid.

12. Creation involves teamwork. In addition, research has become consolidated as a new feature of the culinary creative process.

13. The barriers between the sweet and savoury world are being broken down. Importance is being given to a new cold cuisine, particularly in the creation of the frozen savoury world.

14. The classical structure of dishes is being broken down: a veritable revolution is underway in first courses and desserts, closely bound up with the concept of symbiosis between the sweet and savoury world; in main dishes the "product-garnish-sauce" hierarchy is being broken down.

15. A new way of serving food is being promoted. The dishes are finished in the dining room by the serving staff. In other cases the diners themselves participate in this process.

16. Regional cuisine as a style is an expression of its own geographical and cultural context as well as its culinary traditions. Its bond with nature complements and enriches this relationship with its environment.

17. Products and preparations from other countries are subjected to one's particular style of cooking.

18. There are two main paths towards attaining harmony of products and flavours: through memory (connection with regional cooking traditions, adaptation, deconstruction, former modern recipes), or through new combinations.

19. A culinary language is being created which is becoming more and more ordered, that on some occasions establishes a relationship with the world and language of art.

20. Recipes are designed to ensure that harmony is to be found in small servings.

21. Decontextualisation, irony, spectacle, performance are completely legitimate, as long as they are not superficial but respond to, or are closely bound up with, a process of gastronomic reflection.

22. The menu de dégustation is the finest expression of avant-garde cooking. The structure is alive and subject to changes. Concepts such as snacks, tapas, pre-desserts, morphs, etc., are coming into their own.

23. Knowledge and/or collaboration with experts from different fields (gastronomic culture, history, industrial design, etc.,) is essential for progress in cooking. In particular collaboration with the food industry and the scientific world has brought about fundamental advances. Sharing this knowledge among cooking professionals has contributed to this evolution. 